\title{
A dimensão conceitual da organização do conhecimento no universo científico da ISKO: uma análise de domínio a partir dos congressos da ISKO-Brasil, ISKO-Espanha, ISKO-América Do Norte e ISKO-França
}

La dimensión conceptual de la organización del conocimiento en el universo cientíifico de la ISKO: un análisis de dominio de los congresos de ISKO-Brasil, ISKO-España, ISKO-Norteamerica e ISKO-Francia

The conceptual dimension of knowledge organization in the scientific universe of ISKO: a domain-analysis of ISKO-Brazil, ISKO-Spain, NASKO and ISKO France conferences

\author{
José Augusto Chaves GuimaRÃEs (1), Daniela Fernanda de Oliveira MATOS (2), \\ André Ynada dos SANTOS (3), Rodrigo de SALES (4)
}

(1) (2) (3) Departamento de Ciência da Informação.Universidade Estadual Paulista - Av. Hygino Muzzi Filho, 737 - Marília -SP BRASIL. Correio eletrônico: gpfapoi@gmail.com (4) Departamento de Ciência da Informação. Universidade Federal Fluminense UFF. Rua Tiradentes, 148 - Ingá - Niterói - RJ - BRASIL. Correio eletrônico: rodigo.sales.s@gmail.com

\begin{abstract}
Resumen
Se analiza comparativamente la definición conceptual de la organización del conocimiento y los diferentes referentes teóricos que la sostienen en el ámbito de los congresos realizados por los capítulos brasileño, español, norteamericano y francés de ISKO en sus dos últimos eventos realizados (2011 y 2013). Para ello, se utiliza análisis de contenido y análisis de citas. Los resultados revelan que los cuatro capítulos analizados son bastante prolíficos en el abordaje de la cuestión conceptual del área, aunque ese aspecto todavía se encuentra en construcción. En particular, se detectan dos grandes preocupaciones: por un lado, abordar los aspectos relativos a la naturaleza y al objeto de la organización del conocimiento en su configuración epistemológica, y, por otro, una preocupación de naturaleza más pragmática y operacional, volcada a los procesos e instrumentos de la organización del conocimiento, muchas veces concebidos como objeto del campo. En este contexto, los referentes teóricos predominantes se centran en siete autores (Hjørland; Dahlberg; Gardin; Mai; Olson; Barité y Thelleffssen). Hjørland y Dahlberg asumen un fuerte liderazgo, lo que revela, respectivamente, el énfasis de los abordajes sociocognitivo y ontológico del área, siendo que la primera asume una mayor fuerza en la medida en que especialmente Mai y Thelleffssen, con la cuestión semiótica, y Olson, con los estudios culturales, refuerzan ese cuadro teórico.
\end{abstract}

Palabras clave: Organización del conocimiento. Concepto. Análisis de dominio. Análisis de contenido. Sociedad Internacional para la organización del conocimiento. ISKO. España. Brasil. Francia. América del Norte.

\begin{abstract}
We comparatively analyze the conceptual question of knowledge organization and the theoretical references that are fundamental in the last two Brazilian, Spanish, North American and French ISKO chapter meetings (of 2011 and 2013). From this, we conducted content analysis and citation analysis. The results show that the four analyzed chapters are quite fruitful in addressing conceptual issues, even when this aspect is still in development. This is verified by, on one hand, the concern with aspects such as the nature and object of knowledge organization in its epistemological configuration, and on the other hand, a more pragmatic and operational interest related to knowledge organization processes and tools, sometimes perceived as field objects. In this context, the main theoretical references involve seven authors (Hjørland, Dahlberg, Gardin, Mai, Olson, Barité and Thelleffssen). Hjørland and Dahlberg assume strong leaderships that show, respectively, the emphasis of social-cognitive and ontological approaches in the area. The emphasis of social-cognitive seems to take a greater force especially with Mai and Thelleffssen, with the semiotic question, and Olson, with cultural studies, that reinforce this theoretical framework.
\end{abstract}

Keywords: Knowledge organization. Concept. Domain analysis. Content analysis. International Society for Knowledge Organization. ISKO. Brazil. France. North America. Spain. 


\section{Introdução}

A organização do conhecimento desempenha papel nuclear na Ciência da Informação, apresentando avanço teórico notadamente a partir da criação da ISKO, em 1989. No entanto, observa-se que esse campo ainda se encontra em fase de consolidação, em busca da construção e delimitação de sua própria base conceitual e seus limites, em virtude de distintas influências teóricas. Tal aspecto se revela, dentre outros, por uma diversidade no tocante ao seu aspecto conceitual, notadamente com relação a seu objeto, o que leva à necessidade de analisar de que forma o ambiente científico da ISKO vem construindo/delimitando essa dimensão conceitual, a partir de seu discurso científico oficial em âmbito internacional.

Em pesquisa anterior (Guimarães et al, 2014), relativa ao universo dos congressos internacionais da ISKO, foi possível observar que a organização do conhecimento constitui área ou campo de conhecimento e/ou de aplicação onde, relacionado à construção de discursos especializados. Sua natureza operacional encontra-se ora voltada à organização e representação de conceitos, ora voltada à busca de informação e ao acesso aos conhecimentos, em especial diálogo com a recuperação da informação. Nesse contexto, seu objeto reside na estrutura do conhecimento contida nos documentos (conhecimento registrado, socializado e publicado), com especial ênfase aos conceitos e à sua modelagem assim como às atividades discursivas em domínios científicos e práticas sociais e culturais específicos, a organização do conhecimento vale-se de instrumentos como sistemas de classificação, tesauros, vocabulário e outras linguagens de indexação. Como abordagens predominantes destacam-se as questões relativas a aspectos cognitivos, tecnológicos, lógico-conceituais, socioculturais e de gestão, o que sinaliza para os diálogos mais prementes na área.

Nesta oportunidade, e em continuidade à referida pesquisa, analisa-se comparativamente a questão conceitual da organização do conhecimento nos congressos realizados pelos capítulos brasileiro, espanhol, norte-americano e francês da ISKO nos últimos dois eventos realizados em cada (2011 e 2013) haja vista o fato de o capítulo brasileiro ser ainda recente.

Dessa forma, analisam-se especialmente os referentes teóricos que subsidiam essa dimensão conceitual do campo e até que medida existe diálogo entre essas quatro ambiências analisadas, a partir de seus referentes. Para tanto, vale-se de duas das onze vertentes de análise de domínio propostas por Hjørland (2002): a epistemológica, a partir da análise de conteúdo das definições apresentadas, e bibliográfica, a partir das redes de citação que subsidiam essa produção.

\section{A Organização do Conhecimento e a atuação científica da ISKO}

Embora o termo organização do conhecimento remonte a Henry Evelyn Bliss, na década de 30 do século passado, sua constituição como campo de estudos interdisciplinar é bastante recente, podendo-se atribuir seu nascimento quatro décadas após, quando a partir da década de 70 quando Dagobert Soergel e Ingetraut Dalhberg buscaram conceituá-lo, aspecto que levou à criação, em 1974, na Alemanha, da Society for Classification e, em 1989, da International Society for Knowledge Organization-ISKO.

Para Dahlberg (2008), a Organização do Conhecimento possui natureza científica e destinase a sistematizar unidades de conhecimento (conceitos) a partir dos elementos ou características que Ihes são inerentes. A isso se alia a aplicação de conceitos e de classes de conceitos que possam permitir a transferência de conteúdos (assuntos). Para a autora (Dahlberg, 1993) duas concepções de conhecimento devem ser consideradas: enquanto processo individual, que não é transferível e aquele que pressupõe um consenso, a partir, de um registro, sendo, este último, objeto da organização do conhecimento. Assim, o termo Organização do Conhecimento, para a Ciência da Informação irá se dedicar às atividades de classificar, indexar e representar o conhecimento através de registros com o objetivo específico de suprir a necessidade de informação (Bufrem, 2008).

No âmbito da Ciência da Informação, a organização do conhecimento apresenta natureza mediadora, configurando-se em um conjunto de processos que estabelecem a intermediação entre um conhecimento que, uma vez produzido, foi materializado e socializado, de tal forma que esse conhecimento possa servir de base para a geração de um novo conhecimento. Este novo conhecimento, por sua vez, uma vez materializado e socializado, pode igualmente ser objeto de nova organização e representação caracterizando aquilo que se pode denominar como fluxo helicoidal da informação (Guimarães, 2008).

Tendo por objetivo promover o avanço teórico e aplicado da Organização do Conhecimento em diversos campos e em diferentes maneiras, congregando pesquisadores de distintos países, em áreas como Ciência da Informação, Linguís- 
tica, Filosofia, e Ciência da Computação, entre outras. Desse modo, foram até então realizadas treze conferências internacionais (Darmstadt, 1990; New Delhi, 1992; Copenhagen, 1994; Washington, 1996; Lille, 1998; Toronto, 2000; Granada, 2002; Londres, 2004; Viena, 2006; Montréal, 2008; Roma, 2010; Mysore, 2012; e Cracóvia, 2014), além o que se tem toda uma atuação regional a partir do Germânico, Norteamericano, Francês, Espanhol, Italiano, Brasileiro, Polonês, Escandinavo, Maghreb e Indiano que, por sua vez, também realizam conferências nacionais e/ou regionais.

Nesse contexto, especial destaque merece quatro capítulos que vêm, ao longo do tempo, demonstrado forte produção científica na área bem como um alto nível de preocupação com as questões teóricas concernentes à organização do conhecimento: Espanha, França, América do Norte e Brasil.

A ISKO-Espanha, (hoje transformada em capítulo ibérico para abrigar também Portugal), foi criada em 1990, e possui já onze conferências realizadas (Madrid, 1993; Getafe, 1995 e 1997; Granada, 1999; Alcalá de Henares, 2001; Salamanca, 2003; Barcelona, 2005; León, 2007; Valencia, 2009; Ferrol, 2011; e Porto, 2013).

A ISKO-França A ISKO-França foi criada em 1998 e até o presente momento promoveu nove congressos: Lille, 1998; Lyon, 1999; Paris, 2001; Grenoble, 2003; Nancy, 2005; Toulouse, 2007; Lyon, 2009; Lille, 2011; e Paris, 2013.

A ISKO América do Norte - NASKO, criada em 2007, é formada pelo Canadá e pelos Estados Unidos, tendo realizado até então quatro congressos: Toronto, 2007; Syracuse, 2009; Toronto, 2011 e Milwaukee, 2013.

Por sua vez, o capítulo brasileiro surgiu a partir de um grupo de pesquisadores do Grupo de Trabalho 2: Organização e Representação do Conhecimento, da Associação Nacional de Pesquisa em Ciência da Informação (ANCIB), tendo sido criado em 2007. Até o momento foram realizados dois congressos: Brasília, 2011; e Rio de Janeiro, 2013.

\section{Metodologia}

A pesquisa desenvolveu-se a partir do conjunto de atas dos dois últimos congressos nacionais / regionais realizados (2011 e 2013) pelos capítulos brasileiro, espanhol, norte-americano e francês da ISKO, considerados capítulos de larga produção e que, ao longo do tempo, vêm mantendo certa dialogicidade científica.
A pesquisa centrou-se na abordagem da análise de domínio que, no âmbito internacional da Ciência da Informação, vem sendo tradicionalmente trabalhada, em termos teóricos e aplicados, por Hjorland \& Albrechtsen (1995), Moya Anegón \& Herrero Solana (2001), Hjørland (2002, 2004), Tennis (2003), e Smiraglia (2012), dentre outros, constitui uma importante abordagem para caracterização e avaliação da ciência, na medida em que permite identificar as condições pelas quais o conhecimento científico se constrói e se socializa. Para tanto, tem-se por domínio a concepção de Smiraglia (2012, p.114) enquanto

[...] um grupo que apresenta uma base ontológica que revela uma teleologia subjacente, um conjunto de hipóteses comuns, assim como um consenso epistemológico sobre as abordagens metodológicas, sociais e semânticas a serem utilizadas.

Desse modo, valeu-se de duas das onze abordagens de análise de domínio previstas pro Hjørland (2002): a epistemológica e a bibliométrica. Para tanto, e tendo por base o percurso metodológico utilizado por Guimarães et al. (2014) partiu-se de um levantamento na coleção completa dos referidos Anais, tomando por base a incidência do termo "knowledge organization" (e seus correspondentes em francês, espanhol e português) suas variações em francês, nos títulos, palavras-chave, resumos e títulos de seções das comunicações publicadas. Em seguida, realizou-se a leitura das comunicações que revelaram tal incidência, selecionando-se aquelas que traziam conceitos, definições ou considerações teóricas acerca da natureza da organização do conhecimento.

A dimensão epistemológica foi abordada a partir do universo conceitual da literatura analisada. Dessa forma, no tocante às definições, procedeu-se à Análise de Conteúdo (Bardin, 2009), cuja escolha se justifica pelo fato de seus procedimentos possibilitarem uma análise com base em inferências extraídas de conteúdos de documentos - a partir de uma interpretação controlada por meio de variáveis ou indicadores, que proporcionam maior liberdade ao analista, sem que se perca a objetividade da investigação, e por se tratar de uma análise pautada em definições concebidas por autores/pesquisadores, ou seja, argumentos registrados textualmente. Dividida em três fases: pré-análise, exploração do material e, tratamento dos resultados (inferências e interpretações). Para tanto, a delimitação do corpus respeito as quatro regras básicas previstas por Bardin (2009): exaustividade (na medida em que todas as definições encontradas, quando da coleta de informações, foram consideradas materiais úteis à análise, 
sem processo seletivo); representatividade (assegurada pela escolha do canal de comunicação científica, pois os trabalhos publicados nos Anais da ISKO são de autoria de pesquisadores e teóricos que representam uma parcela significativa da produção internacional em organização do conhecimento); homogeneidade (pois as definições analisadas são relativas a um mesmo tema, assim, são homogêneos tanto na estrutura textual quanto no assunto); e pertinência do material (as fontes são as próprias ideias explicitadas pelos autores/pesquisadores em suas definições).

As definições foram analisadas a partir das seguintes categorias: natureza, objeto, objetivo, instrumentos, processos, diálogos interdisciplinares e perspectivas ou abordagens.

Em seguida, procedeu-se à análise bibliométrica das fontes utilizadas nos referidos artigos de modo a identificar os referentes teóricos predominantes e as interlocuções estabelecidas entre os capítulos.

Para tanto, foram construídas e analisadas as redes de citação, valendo-se, para tanto do software PAJEK (2009).

\section{Apresentação, análise e discussão dos resultados}

Partiu-se de um conjunto de 85 trabalhos na ISKO-Brasil, 132 na ISKO-Espanha, 43 na NASKO e 45 na ISKO-França, totalizando 305 trabalhos. Desse conjunto, e a partir da incidência dos termos no título, chegou-se a um grupo de 33 trabalhos na ISKO-Brasil, 57 na ISKOEspaña, 21 na NASKO e 9 na ISKO-França, totalizando 120 trabalhos. Em seguida, procedeu-se à leitura desse material, observando-se que nem todos traziam, efetivamente, conceituações, definições ou considerações teóricas sobre a organização do conhecimento. Dessa foram, chegou-se a um corpus final de 16 trabaIhos na ISKO-Brasil, 17 na ISKO-Espanha, 10 na NASKO e 5 na ISKO-França, totalizando 48 comunicações.

Considerando o fato de que todos os autores das comunicações publicadas nos Anais da ISKO são pesquisadores e ou teóricos da área, cujas concepções contribuem efetivamente para a construção do universo epistemológico da organização do conhecimento em âmbito internacional, optou-se por nomeá-los em suas definições. Dessa forma, o universo dos autores das definições aqui levantadas é composto pelo seguinte conjunto de autores: a) NASKO: MacCall, 2011; Fox, 2011; Pattuelli, 2011; Adler \& Tennis, 2013; Fox, Reece, 2013; Frické, 2013;
Jacob, Zhang, 2013; Marchese \& Smiraglia, 2013; Olson \& Howarth, 2013; Sales \& Guimarães, 2013; Tognoli, Guimarães \& Tennis, 2013; Schatz, Stennes-Spidahl \& Loehrlein, 2013; b) ISKO-França: Arsenault \& El Hadi, 2011; Couzinet, 2011; Gnoli, 2011; Sosinska-Kalata, 2011; c) ISKO-Brasil: Andrade, Berti Junior, Cervantes, Rodrigues, 2011; Miranda, Paranhos, Oliveira, Paes, 2011; Dodebei , 2011; Brascher, 2011, 2013; Ohly, 2011; Barite, 2011; Smiraglia, 2013; Alvarenga, 2013; Fujita, 2013; Varela \& Barbosa, 2013; e d) ISKO-Espanha: AlfayaLamas, 2011; Sanchéz, Blázquez, Rodríguez, 2011; Guimarães, Ferreira, Freitas, 2011; Tognoli, Milani, Barros, 2011; Café, Agustín-Lacruz, Barros, 2011; Ohly, 2011; Almeida, 2013; Alvares Júnior, Saldanha, 2013; Dal'evedove, Almeida, Fujita, 2013; Martins, Moraes, 2013; Murguia, Sales, 2013; Semidão, Almeida, Moreira, 2013.

Aplicando-se a análise de conteúdo ao referido corpus, obtiveram-se os seguintes resultados:

\subsection{ISKO-Brasil}

Segundo a análise feita nos artigos publicados nos Anais da ISKO-Brasil, o objeto da O.C. está relacionado à classificação conceitual, à teoria, indexação e representação/terminologia de conceito (Andrade, Junior, Cervantes, Rodrigues, 2011; Ohly, 2011b; Fujita, 2013). Para alguns autores (Miranda, Paranhos, Oliveira, Paes, 2011) seu objeto são os fundamentos científicos e o desenvolvimento das técnicas de planejamento, construção, uso, gestão e avaliação das habilidades e ferramentas empregadas nos sistemas de informação para tratamento, armazenamento e recuperação de documentos. Guimarães (2001) e Barité (2009) entendem o conhecimento socializado ou registrado, o saber acumulado que se documenta por meio dos procesos de publicação, edição, registro em patentes ou em formas de socialização do saberes similares como objeto da O.C. Tal definição está relacionada à informação como conhecimento (information-as-knowledge) e à informação como coisa (information-as-thing), duas aproximações à noção de "conhecimento materializado" e registrado (Barite, 2011). Segundo Smiraglia (2013) o objeto da O.C. é uma comunidade de discurso onde uma investigação rigorosa, autoconsciente acontece em relação àquilo que é conhecido, e suas várias ordenações e sequencias, tanto as naturais ou heurísticas, e aquelas que são impostas. Para Brascher (2013) as representações de domínios do conhecimento são objeto de estudo da O.C. 
Com relação aos objetivos os processos de representação, organização e recuperação ganham um destaque importante a partir das definições levantadas. Para Andrade, Junior, Cervantes, Rodrigues (2011) o objetivo da O.C é possibilitar a recuperação de objetos e conteúdos informacionais nos ambientes digitais, bem como estruturar e arranjar sistematicamente unidades do conhecimento (os conceitos) de acordo com os elementos de conhecimento (Andrade, Junior, Cervantes, Rodrigues, 2011; Ohly, 2011b). Ainda segundo Ohly (2011b) os objetivos da O.C são organizar: a) unidades de conceitos de conhecimento e b) todos os tipos de objetos (minerais, plantas, documentos, pinturas, objetos de museus, etc), relacionados a termos ou categorias particulares, para capturar o que é conhecido sobre o mundo em alguma forma ordenada permitindo seu futuro compartiIhamento com outros. Ortega (2013) advoga a favor da promoção do acesso ao conhecimento, visando seu uso e nova produção bem como elaborar representações que possam ser significadas e manipuladas a favor de um certo público. Ainda segundo a autora a organização do Conhecimento deve ser capaz de fornecer respostas à sociedade para o problema do crescimento dos documentos, por meio de critérios científicos, como uma extensão da capacidade de organização e transferência daquilo que o ser humano sabe. Para Alvarenga (2013), a O.C deve prover uma estrutura ou esquema para armazenar e organizar dados, informação ou conhecimento sobre o mundo e sobre pensamentos, visando à compreensão, recuperação ou descoberta, ao raciocínio e a outros propósitos. Segundo Brascher (2013), delimitar o significado de termos no contexto de seus domínios e estabelecer relações conceituais que auxiliam a posicionar um conceito no sistema conceitual são os objetivos da Organização do Conhecimento. E, por último, Miranda, Paranhos, Oliveira, Paes (2011) definem a geração de um novo conhecimento como objetivo final da O.C.

Os processos apresentam já um maior consenso entre os autores pesquisados, uma vez que a descrição, indexação e classificação ou representação figuram entre os processos nucleares da O.C. Segundo Barité (2001) esses processos consistem na construção, desenvolvimento e gestão de sistemas de organização do conhecimento e outras estruturas conceituais, e nas atividades de classificação e indexação para a recuperação temática de documentos, recursos, dados e todo tipo de informação. Para Fujita (2013) os processos também são a descrição documentária, indexação e classificação, conceitos ligados à representação da informação apontados como processos por Varela e Barbo- sa (2013). Ainda segundo os autores, a criação de recursos mediadores do acesso ao conhecimento também é entendida como um processo da O.C.

Como instrumentos, Andrade Junior, Cervantes, Rodrigues (2011) apontam a linguagem do sistema de informação (controlada).

No tocante à natureza da OC encontramos, ainda, mais divergência. Enquanto alguns a caracterizam como ciência (Andrade, Junior, Cervantes, Rodrigues, 2011; Ohly, 2011b) ou disciplina científica (Miranda, Paranhos, Oliveira, Paes, 2011), outros a definem como área temática (Ohly, 2011), domínio (Smiraglia, 2013), conjunto de procedimentos e ferramentas que ajudam os seres humanos a compreender $\mathrm{e}$ ordenar o caos cognitivo (Ortega, 2013), atividade-meio para o objetivo ou fim da $\mathrm{Cl}$ (Ortega, 2013) e de caráter social, cognitivo e mediador (Varela, Barbosa, 2013).

Seus diálogos interdisciplinares apresentam as novas tecnologias (Brascher, 2011, Ortega, 2013), a fundamentação teórica à Indexação (Fujita, 2013) e as inter-relações de pesquisa com domínios além dos tradicionalmente contemplados (Brascher, 2011), ultrapassando barreiras disciplinares permitindo a fertilização de outras áreas do conhecimento (Dodebei, 2011).

\subsection{ISKO - Espanha}

Nos Anais da ISKO-Espanha é possível perceber uma divisão de autores que caracaterizam a Organização do conhecimento, quanto a sua natureza, como disciplina científica aplicada que ainda carece de uma exploração mais efetiva de sua base teórica (Sanchéz, Blázquez, Rodríguez, 2011; Guimarães, Ferreira, Freitas, 2011; Miranda, 2007; Ohly, 2011a), e outra divisão que a caracteriza enquanto ciência (AlfayaLamas, 2011; Ohly, 2011a) (Café, AgustínLacruz, Barros, 2011) (Semidão, Almeida, Moreira, 2013). Sua compreensão enquanto um campo de estudos relacionado à informação também se faz presente em algumas obras (Alfaya-Lamas, 2011; Dal'Evedore, Almeida, Fujita, 2013; Almeida, 2013). Outros autores possuem uma visão única e diferenciada da natureza da O.C. Para Café, Agustín-Lacruz, Barros (2011) é uma área de estudos interdisciplinar, enquanto para Semidão, Almeida e Moreira (2013) é uma área temática. Para Tognoli, Milani e Barros (2011) a O.C é um marco teórico-conceitual. Para Almeida (2013), ela é tanto uma atividade quanto um fenômeno. Martins e Moraes (2013) a consideram um processo de modelagem conceitual enquanto Murguia e 
Sales (2013) acreditam que a O.C seja um fazer de natureza operacional.

O objeto de estudo da OC, segundos as definições encontradas nos anais da ISKO-Espanha, está ligado basicamente ao processo de organização. Assim, para Alfaya-Lamas (2011), Café, Agustín-Lacruz, Barros (2011), Ohly (2011a), Semidão, Almeida, Moreira (2013) o objeto da O.C é o arranjo estrutural e sistemático das unidades de conhecimento (conceitos) segundo elementos de conhecimento inerentes (características) e a aplicação de conceitos e clases de conceitos ordernadas para a atribuição dos conteúdos de referências de todos os tipos que valem a pena conhecer. Para Murguia e Sales (2013) é a organização de unidades de conhecimento ou organização de conceitos e para Guimarães, Ferreira, Freitas (2011) é a organização da informação em registros bibliográficos, incluindo índices de citação, texto completo e internet. Segundo Alfaya-Lamas (2011) seu objeto é a natureza e qualidade dos procesos de organização do conhecimento e dos sistemas de organização do conhecimento. Para Tognoli, Milani, Barros (2011) é o conhecimento registrado e socializado. Para Almeida (2013) são os objetos existentes que impactam diretamente a vida de cientistas, das pessoas e das comunidades envolvidas e, por último, para Murguia e Sales (2013), o objeto de estudo da OC são os conceitos e estruturas conceituais formalizadas instrumentalmente em sistemas de organização do conhecimento, como sistemas de classificação, tesauros e ontologias.

O objetivo da O.C está fundamentalmente ligado às questões referentes à recuperação a acesso à informação registrada, como demonstram as definições a seguir. Jiménez (2011) e Alfaya-Lamas (2011) priorizam o acesso e a recuperação à informação, enquanto Semidão, Almeida, Moreira (2013) focam o armazenamento eficiente, recuperação e processamento da informação codificada em texto e em outros artefatos humanos. A organização e representação do conhecimento também são objetivos que estão presentes nas definições de AlfayaLamas (2011), Martins e Moares (2013).

No tocante aos diálogos interdisciplinares, as novas tecnologías e a inteligencia artificial se fazem presente no texto de Sanchéz, Blázquez, Rodríguez (2011). OS diálogos com a Biblioteconomia também estão presentes nos textos de Ohly (2011a) e Álvares Júnior e Saldanha (2013). A linguística e a teoria da classificação também são apresentadas por Garcia Marco como diálogos com a O.C. Bufrem, Breda, Silva e Fecchio (2013) destacam as relações com a gestão do conhecimento e inteligencia competi- viva. Almeida (2013) destaca que há uma necessidade do contato com outras disciplinas para a execução de seus produtos e processos.

Os processos estão fundamentalmente ligados à classificação, indexação, catalogação e representação do conhecimento, bem como à elaboração de resumos (Alfaya-Lamas, 2011; Guimarães, Ferreira, Freitas, 2011; e Tognoli, Milani, Barros, 2011), e ao tratamento temático da Informação (Murguia, Sales, 2013).

\subsection{ISKO - França}

Analisado os anais da ISKO-França, nota-se falta de consenso quanto à natureza da OC. Enquanto Gnoli (2011) a considera uma disciplina (em construção), Couzinet (2011) atenta para sua ligação à organização social do conhecimento, como um domínio que visa a constituir-se em uma ciência situada entre a indexação e a comunicação científica. No entanto, no tocante ao objeto, tem-se a abordagem do conhecimento (Gnoli, 2011; Arsenault \& El Hadi, 2011) ou ainda o documento (Gnoli, 2011). Os objetivos da OC, por sua vez, estão ligados à atribuição de sentido, à recuperação, ao acesso e à apropriação da informação (Couzinet, 2011; Sosinska-Kalata, 2011).

Os processos da área encontram-se ligados a indexação, classificação e análise de conteúdo (Arsenault \& El Hadi, 2011; Couzinet, 2011) sendo que os instrumentos de OC centram-se nas linguagens documentais (Couzinet, 2011) e as ferramentas dependentes da identificação e da representação de numerosas características formais e do conteúdo informacional dos recursos documentários (Sosinska-Kalata, 2011).

Ós diálogos intedisciplinres da área vão além da Ciência da Informação, encontrando um espaço de interlocução com a Informática, filosofia, linguística, as ciências cognitivas, as ciências da educação e mais geralmenre as ciências humanas e sociais (Couzinet, 2011).

\subsection{NASKO}

Assim como nos demais capítulos analisados, não se verifica, nos anais da NASKO, um consenso no que tange à natureza da Organização do Conhecimento, pois enquanto Dousa (2009) e Smiraglia (2009) a consideram um subcampo da ciência e da Ciência da Informação e Bibliotecomia, Sales \& Guimarães (2013) a consideram um domínio de natureza predominantemente operacional.

Não se observam abordagens relativas aos objetivos da OC, sendo que o objeto da área estaria nas estruturas conceituais que compõem 
os Sistemas de Organização do Conhecimento, tais como os sistemas de classificação, tesauros e ontologias (Sales, Guimarães, 2013). Já os instrumentos da OC são caracterizados como os vocabulários controlados (Pattuelli, 2011). Os processos, por sua vez, apresentam-se invariavelmente ligados à descrição, indexação e classificação de documentos de arquivo e bibliotecas. (Tognoli, Guimarães, Tennis, 2013).

Relativamente aos diálogos interdisciplinares, são citadas a Arquivística, a Ciência da Informação e a Bibliotecomonia (Tognoli, Guimarães, Tennis, 2013) e Lógica (Frické, 2013).

\section{Análise geral}

De uma análise geral dos quatro capítulos analisados tem-se que a natureza da organização do conhecimento é ainda objeto de profícua discussão na área, na medida em que se verifica, por um lado, o reconhecimento de seu marco teórico-conceitual de modo a posibili-tar sua classificação como ciência, disciplina científica (alguns destacando seu caráter aplicado e o fato de ainda se encontrar em construção), como dominio ou como área ou campo de de estudos interdisciplinar ou ainda como subcampo da ciência e da Ciência da Informação e Biblioteconomia. Por outro lado, alguns autores destcam sua natureza prática, como atividade-meio, conjunto de procedimentos e ferramentas, fazer de natureza operacional ou processo de modelagem conceitual.

Relativamente ao objeto, abordado pelos quatro capítulos da ISKO analisados, preponderam as concepções relativas ao conhecimento, materializado, resgistrado ou socializado em documentos, assim como as unidades de conhecimento: conceitos e sistemas de conceitos. Considerando o campo em si, alguns autores centram o objeto nos fundamentos científicos, nas técnicas e na comunidade discursi-va que o caracterizam. Por outro lado, e em uma dimensão mais pragmática, há autores que reconhecem o objeto do campo nos seus processos (organização, representação, indexação, classificação) ou nos seus instrumentos (sistemas de classificação, tesauros, ontologías).

No que tange aos objetivos, observa-se que apenas o capítulo norte-americano a eles não faz menção. Observa-se uma forte ênfase nos procesos de representação, armazenamento, arranjo, recuperação, acesso e apropriação da informação, por meio do estabelecimento de relações conceituais que promovam a atribuição de sentido para fins de geração de novo conhecimento. Esses processos são efeti-vamente abordados nos quatro capítulos analisados, encontrando-se diretamente ligados à questão do conteúdo ou tratamento temático. Nesse contexto, a indexação, a classificação, a descrição e a condensação são mencionadas como procesos de representação (da informação e do conhecimento), ao que se aliam as preocupações com a construção, desenvolvimento e gestão de sistemas de organização do conhecimento (SOS).

Os instrumentos / ferramentas para desenvolvimento dos processos são abordados pelos capítulos brasileiro, francês e norte-americano, sendo identificados como linguagens controladas dos sistemas de infromação, vocabulários controlados ou linguagens documentais.

Os diálogos interdisciplinares, observados com maior ênfase nos capítulso francés e espanhol, são abordados no contexto da Ciência da Informação (notadamente com a arquivística e a bibliotecomonia) e, no contexto geral das demais ciências humanas e sociais, tais como as ciências cognitivas, a educação, a filosofía, a gestão do conhecimento, a lingüística e a lógica. Destaca-se, ainda, a conexão com a dimensão tecnológica (informática, inteligencia artificial e nova tecnologías em geral).Em seguida, e de modo a caracterizar o domínio, realizou-se uma análise bibliométrica, mais especificamente no que se refere à análise de citações, a partir das fontes (referentes teóricos) utilizadas pelos trabalhos selecionados, de modo a identificar dialógicos, consonâncias correntes teóricas, etc.

Por meio da análise de citações torna-se possível analisar o impacto e a visibilidade de autores em um dado domínio científico, sendo especialmente útil para que se identifiquem escolas teóricas. Dessa forma, constitui especial ferramenta para que, a partir de análise qualiquantitativas, se possa melhor compreender o universo epistemológico de um dado domínio, uma vez que a frequência de cocitação entre dois autores, além de evidenciar aquilo que Small (2004, p.71) denomina como "padrões de co-reconhecimento" determina a proximidade de conteúdos e de como a estrutura de conhecimento de uma área é percebida por seus pesquisadores. Assim, pode-se detectar tanto a similaridade quanto a contraposição de ideias (Vanz, Caregnato, 2003; Gmür, 2003).

A partir desses estudos torna-se possível a construção e visualização gráfica de redes sociais de citação e de cocitação, especialmente útil na visualização do comportamento de um dado domínio de conhecimento (Small, 2004; Guimarães et al., 2011). 
Para fins de visualização das redes de citação, utilizou-se do software PAJEK, descrito por Adamic (2008).

As análises de citações relativas aos trabalhos que trouxeram definições de organização do conhecimento desconsideraram as autocitações e as entidades coletivas, cehgando-se ao seguinte resultado, como se descreve abaixo.

No caso da ISKO-Brasil, teve-se um conjunto de 110 autores citados em 541 citações, dos quais apenas $15(14,4 \%)$ obtiveram um mínimo de duas citações. Na ISKO-Espanha, teve-se um conjunto de 348 autores citados em 147 citações, dos quais apenas $72(20,7 \%)$ obtiveram um mínimo de duas citações. No caso da NAS$\mathrm{KO}$, foram 426 autores citados em 610 citações, dos quais $264(42,8 \%)$ obtiveram um mínimo de duas citações. A ISKO-França, por sua vez, contribuiu com 118 autores, citados em 147 referências, sendo que 43 autores $(29,3 \%$ do total) obtiveram ao menos duas citações.

Para fins de corte para análise em redes de citação, aplicou-se a Teoria do Elitismo de Price, segundo a qual:

A distribuição da produtividade dos autores numa coordenada cartesiana é uma distribuição tão inclinada que inspirou Price (1963) a propor a Lei do Elitismo. Segundo esta lei, se k representa o número total de contribuintes numa disciplina, representaria a elite da área estudada, assim como o número de contribuintes que gera a metade de todas as contribuições (Urbizagástegui Alvarado, 2008, p.1).

Desse modo, relativamente a ISKO-Brasil, em 16 artigos, com 110 autores citados, o corte recaiu sobre um conjunto de 15 autores citados que receberam um mínimo de 2 citações. No caso da ISKO-Espanha, em 17 artigos, com 233 autores citados, o corte recaiu sobre um grupo de 44 autores citados que receberam um mínimo de 3 citações. Na ISKO-França, em 4 artigos, com 68 autores citados, o corte recaiu sobre 6 autores citados, que receberam um mínimo de 2 citações. Já no caso da NASKO, em 10 artigos, com 286 autores citados, o corte recaiu sobre um grupo de 15 autores citados, que receberam um mínimo de 3 citações. De modo a elucidar essa situação apresentam-se, em apêncide, as quatro redes de citação dos capítulos analisados.

No caso brasileiro (Figura 1), dois referentes teóricos internacionais despontam com forte proeminência: Dahlberg e Hjorland, na medida em que recebem citações de diversos autores citantes. Dentre os nacionais, registram-se mais especialmente Smit, Pinho e Guimarães. Destaque-se ainda o fato de que o universo de citados se compõe de um conjunto de autores de forte presença no cenário da ISKO em âmbito internacional, tais como Beghtol, Fugmann, Garcia Gutierrez e Mai.

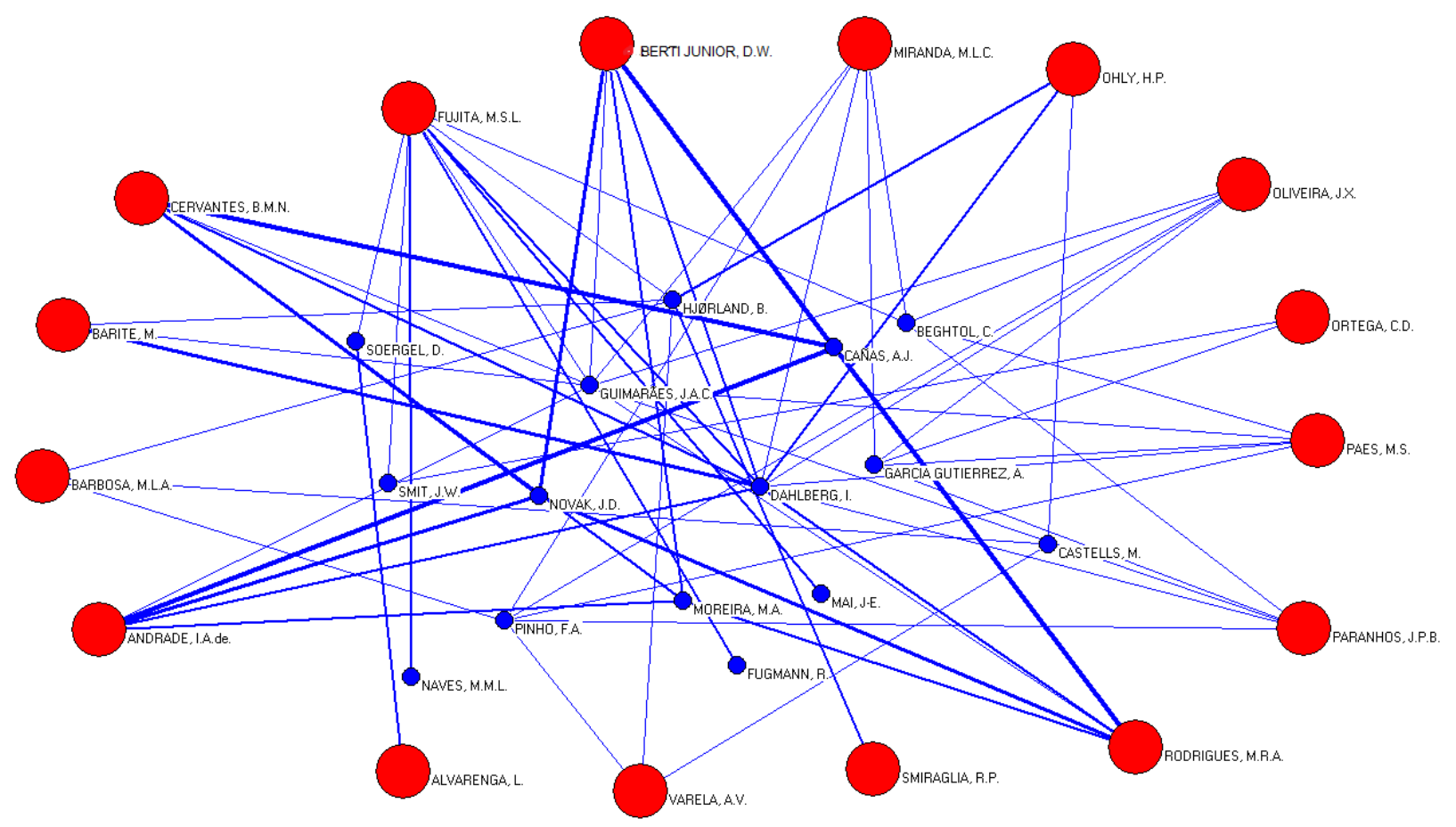

Figura 1. Rede de citação da ISKO-Brasil (círculos vermelhos: citantes; círculos azules: citados) 
Relativamente à rede do capítulo español (e hoje ibérico) (Figura 2), da ISKO, mantém-se a proeminência de Dahlberg e Hjorland. Nesse contexto, observa-se a presença de um mais amplo número de referentes teóricos internacionais da área, como Albrechtsen, Barité, Capurro, Gardin, Olson, Peirce, Pombo, Rayward, Thellefssen e Zeng. A esse se aliam três refe- rentes brasileiros (Fujita, Santaella e Souza). No âmbito español destacam-se Izquierdo Arroyo e López Huertas.

A rede francesa (Figura 3 ), reitera o nome de Hjorland assim como o de Beghtol e acrescenta como referentes internacionasi os nomes de Hartel, Svenonius e Szostak, assim como, no ámbito francés, vale-se de Meyriat.

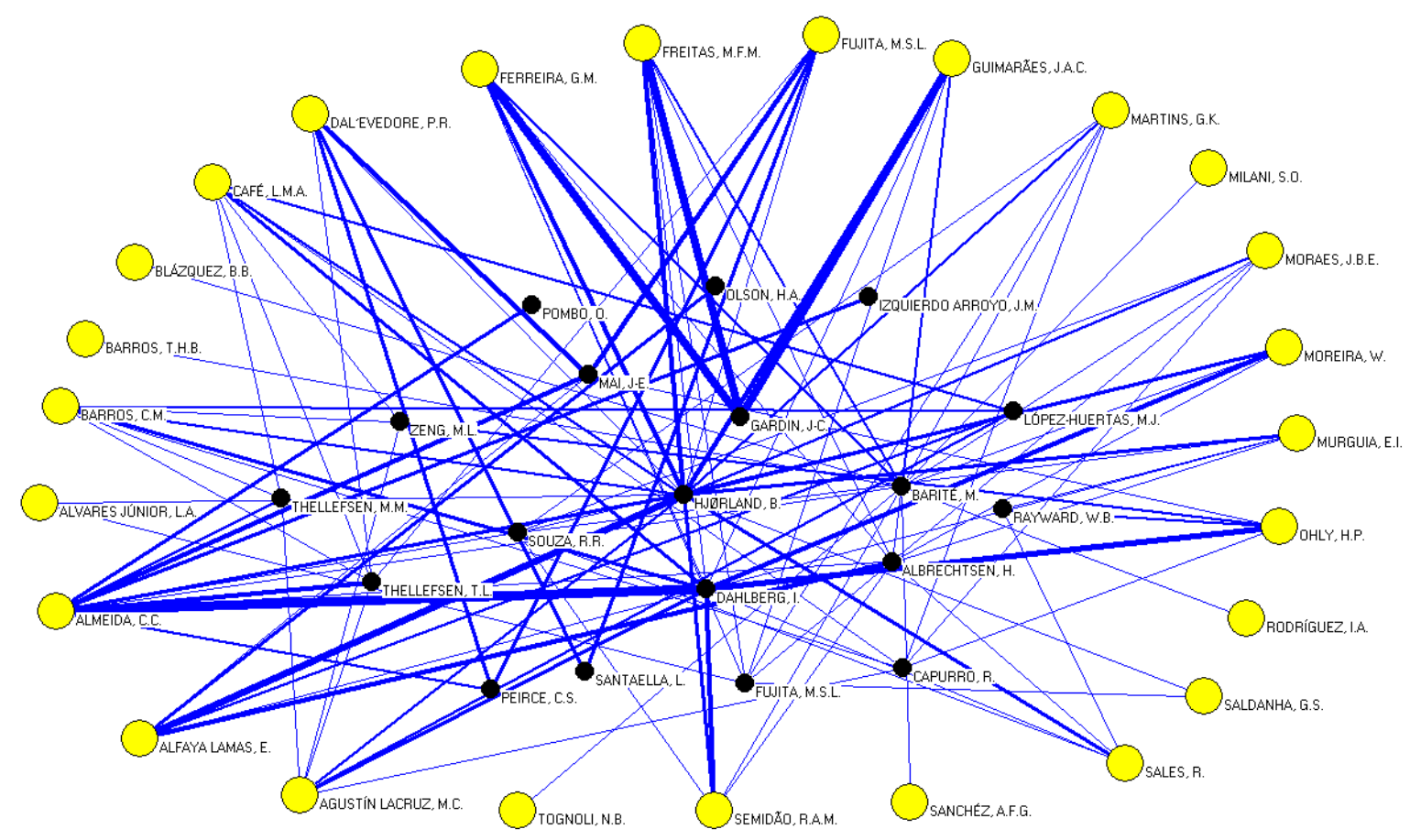

Figura 2. Rede de citação da ISKO-España (círculos amarelos: citantes; círculos pretos: citados)

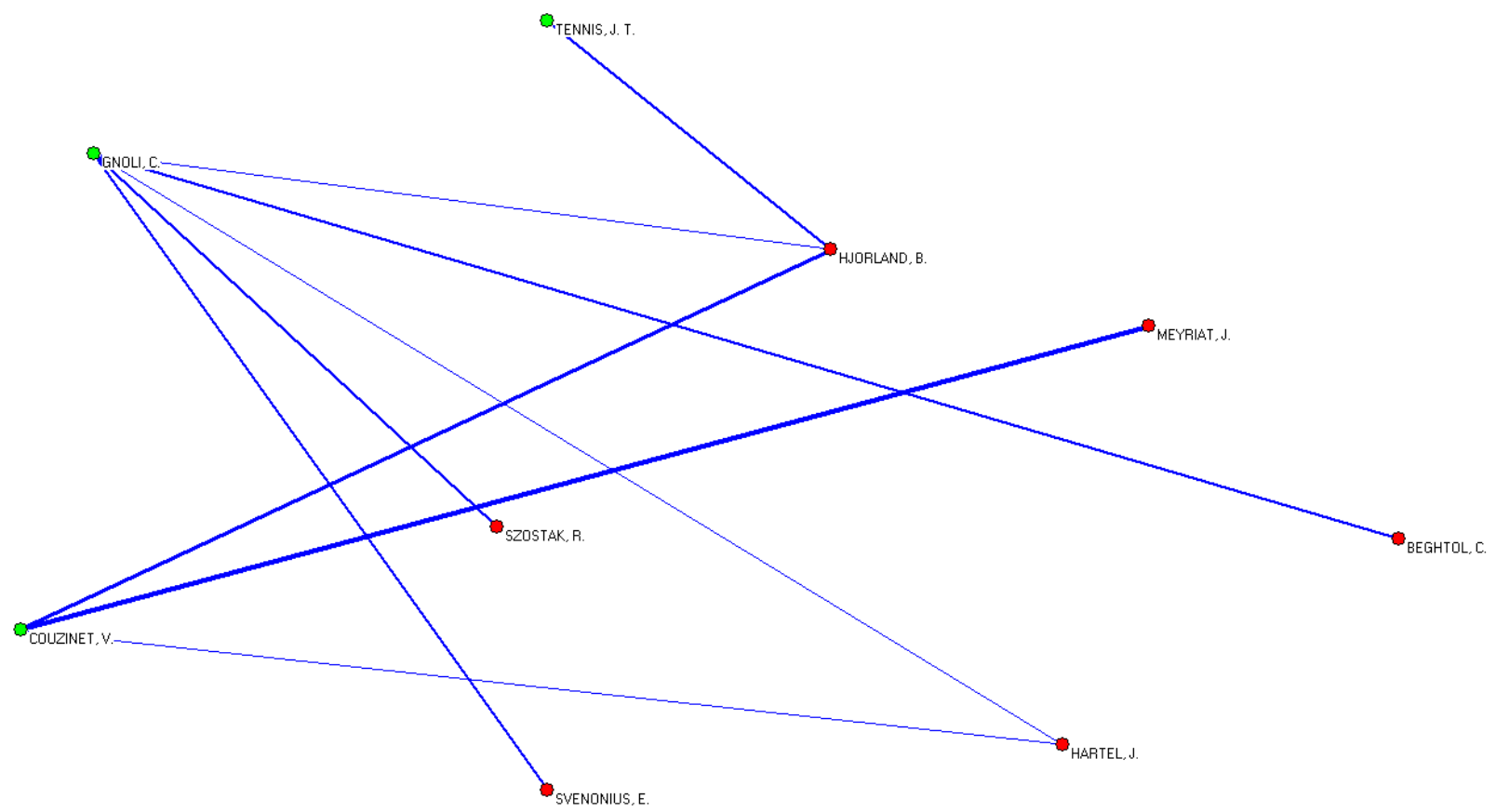

Figura 3. Rede de citação da ISKO-França (verde: autores citantes; vermelho: autores citados) 


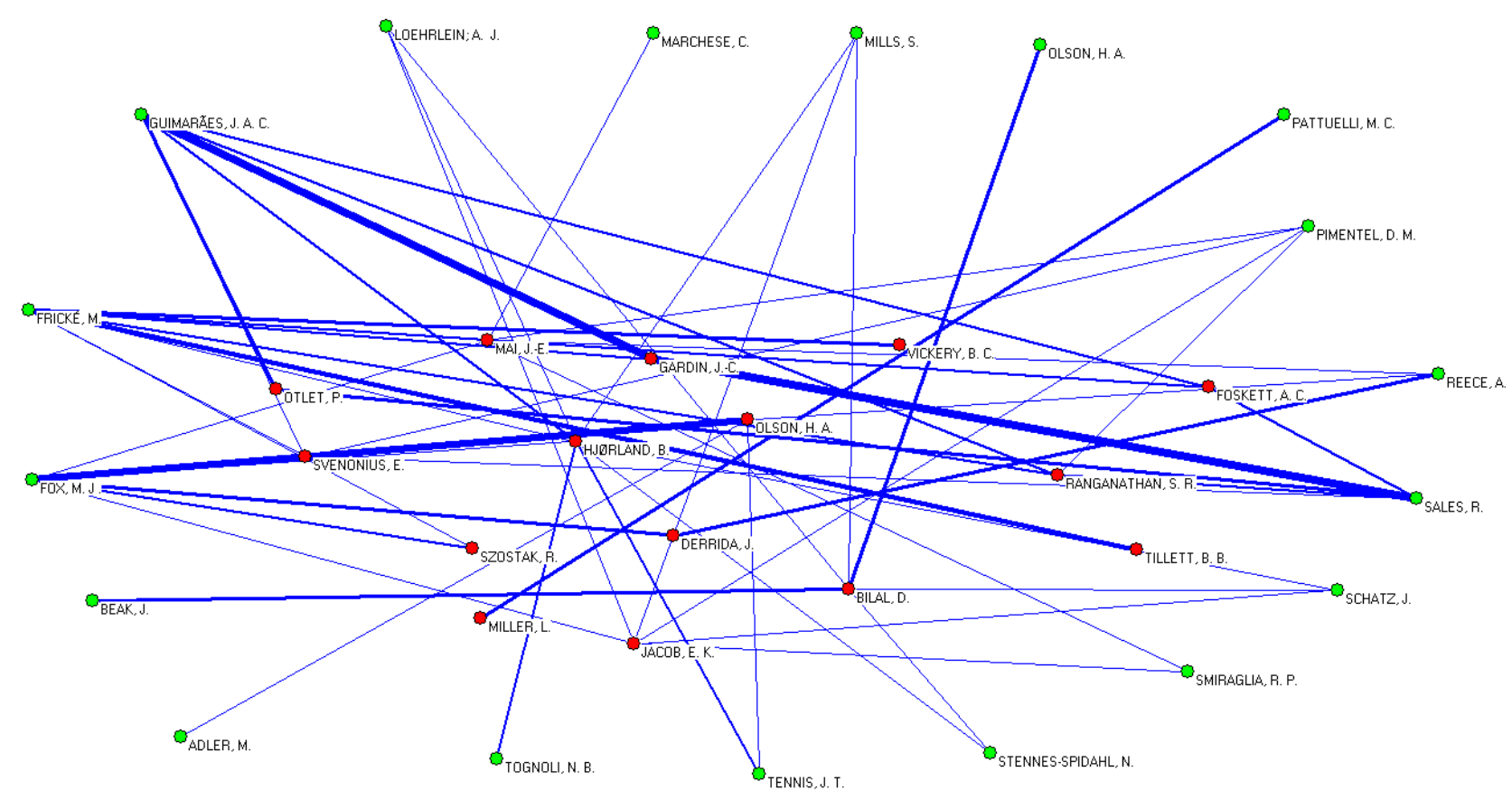

Figura 4. Rede de citação da NASKO (verde: autores citantes; vermelho: autores citados)

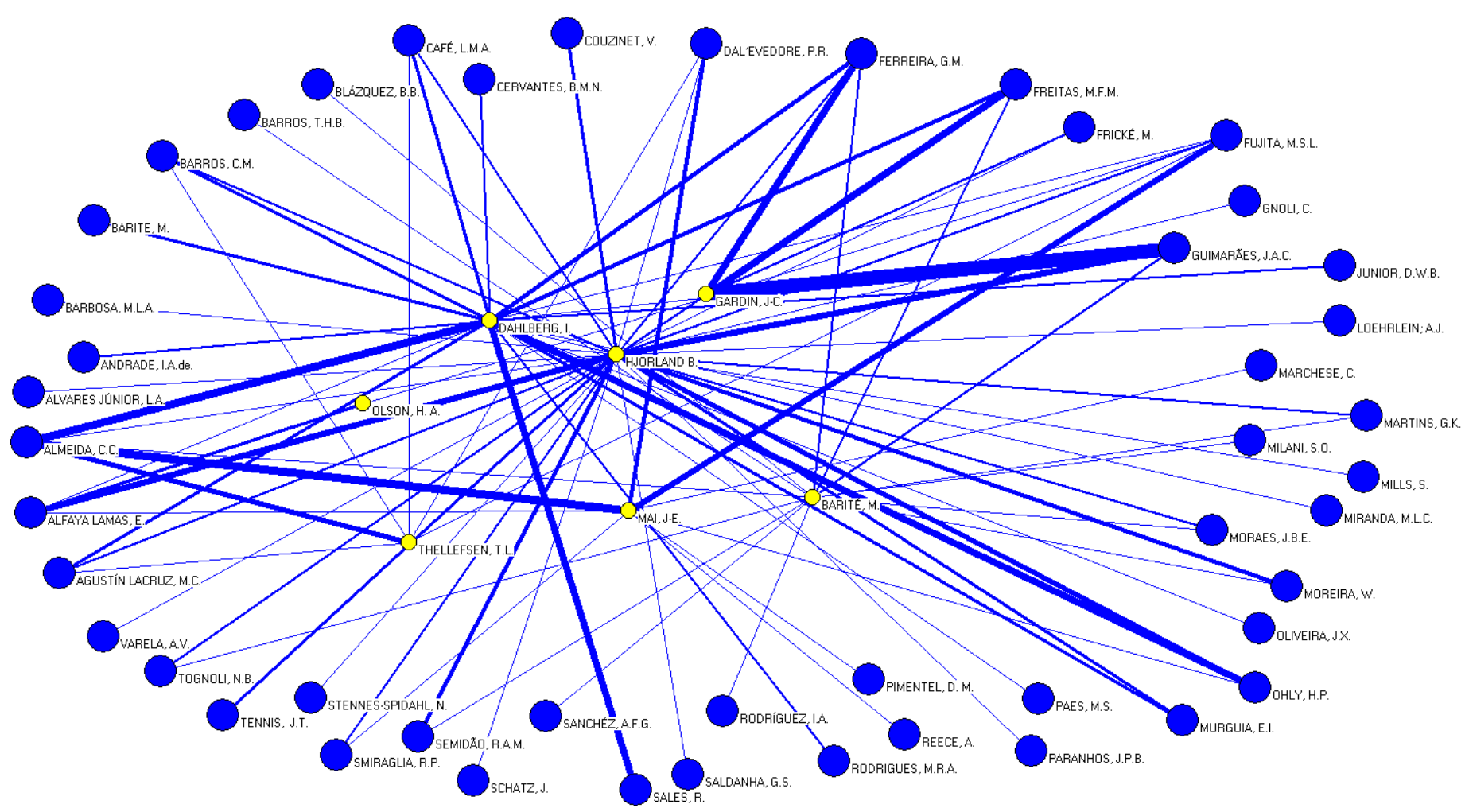

Figura 5. Rede de citação da ISKO: España, Brasil, França e NASKO (círculos azules: citantes; círculos amarelos: citados)

No contexto norte-americano (Figura 4), verificase uma maior índice de citações de autores daquela região se comparados à realidade dos demais capítulos analisados (Bilal, Jacob, Miller,
Olson e Svenonius). No ámbito internacional, destacam-se os francófonos Derrida, Gardin e Otlet, os ingleses Foskett e Vickery, o dinamarqués Mai e o indicano Ranganathan. É intere- 
sante observar que essse é o único capítulo emq ue a presença de Hjorland e de Dahlberg não se faz notar de maneira mais efetiva.

De uma análise geral, Hjørland desponta como o referente mais compartilhado no contexto analisado, na media em que se encontra presente em três dos quatro capítulos (Brasil, Espanha e França). Em seguida, destacam-se seis outros autores que são citados em dois dos quatro capítulos analisados, a saber: Beghtol (Brasil e França), Dahlberg (Brasil e Espanha), Gardin (Espanha e NASKO), Mai (Brasil e NASKO), Olson (Espanha e NASKO) e Svenonius (França e NASKO). Considerando esse conjunto de autores mais citados, observa-se uma forte presença das abordagens sóciocognitivas (Hjorland, Beghtol, Olson, Mai), o que parece sinalizar para uma tendencia investigativa do campo de organização do conhecimento.

De modo a analizar a questão dos referentes teóricos a partir de uma dimensão global dos quatro capítulos analisados, chegou-se, a partir das redes individuais de cada capítulo, a um conjunto geral de 44 autores mais citados, o que, pela aplicação da Teoria de Elistimo de Price, levou a um núcleo de sete autores citados, com um mínimo de seis citações cada (Hjørland, com 45 citações; Dahlberg com 33; Gardin com 17; Mai com 15; Olson com 10; Barité com 8; e Thellefsen com 6) que integram a rede geral (Ver Apêndice - Figura 5).

A análise da referida rede reafirma a centralidade de Hjorland no universo científico da organziação do conehcimento, pois além de ser o autor mais citado, é aquele que recebe citações de uma maior diversidade de autores citantes. Em termos de vinculações teóricas, manifestadas pelas relações citante-citado, observa-se um forte nexo de Guimarães com as ideias de Gardin, de Ohly com as ideias de Dahlberg e de Almeida com as ideias de Mai, o que reflete, respectivamente, as dimensões temáticas relativas à análise documental, a teoría do conceito e aos estudos semióticos em organização do conhecimento.

\section{Conclusão}

A vista dos resultados obtidos, pode-se observar que os quatro capítulos da ISKO analisados (Brasil, Espanha, França e Norte-América) são bastante profícuos na abordagem da questão conceitual da área. No entanto, essa questão ainda se encontra em construção na medida em que se tem por um lado, os aspectos relativos à natureza e ao objeto da organização do conhecimento revelam uma preocupação com a sua configuração epistemológica, voltada para a sua configuração como ciência, domínio ou campo centrado em um conhecimento produzido pela sociedade e por ela materializado para fins de permanencia no tempo e portabilidade no espaço. Por outro lado, uma preocupação de natureza pragmática e operacional se coloca, voltada para os processos os instrumentos da organização do conhecimento, muitas vezes concebidos como seu proprio objeto.

No tocante aos referentes teóricos, observa-se uma forte diversidade - e complementaridade entre os países, o que reforça a assertiva de uma divesidade de abordagens típica de um campo ainda em construção. Sete autores, nesse universo, revelam centralidade (Hjørland, Dahlberg, Gardin, Mai, Olson, Barité e Thelleffssen). Hjørland e Dahlberg assumem forte liderança, o que revela, respectivamente, duas abordagens teóricas da organização do conhecimento: a sócio-cognitiva e a ontológica, em que a primeira assume maior força na medida em que especialmente Mai e Thelleffssen, com a questão semióitca e Olson, com os estudos culturais, vêm se somar a esse quadro teórico.

Por fim, observa-se que a questão conceitual constitui tema relavante para o desenvolvimento da organização do conhecimento especialmente quando distintos capítulos da ISKO são criados e se consolidam, para que se possa pensar em traços distintivos mínimos que permitam a caracterização desse campo como tal.

\section{Referências}

Adamic, L. (2008). Introductory social network analysis with Pajek. Ann Arbor (MI): School of Information, University of Michigan, 2008.

Adler, Melissa; Tennis, Joe (2013). Toward a Taxonomy. // The Fourth North American Symposium on Knowledge Organization - NASKO, 2013: Transition Cultures, Transition KO: Evolving Exploration, Critical Reflection, and Practical Work, Milwaulkee. Proceedings from North American Symposium on Knowledge Organization. Milwaukee: University of Wisconsin-Milwaukee, 2013, 1-19.

Alfaya Lamas, E. (2011). La asunción del género neutro en la teoría y práctica de la organización del conocimiento. // Péres Pais, Maria Carmem; Bonome, María G. (ed.). Actas del X Congreso de ISKO-ESPANHA - 20 years of ISKO Spanish Chapter. Universidad da Coruña - Serviço de Publicaciones, 2011, 249-263.

Almeida, C.C. (2013). Notas sobre os conceitos Semióticos fundamentais á Organização do Conhecimento. // Ribeiro, Fernanda; Cerveira, Maria Elisa. (ed.). Informação e/ou Conhecimento: as duas faces de Jano. Porto: Faculdade de Letras da Universidade do Porto/ CETAC.MEDIA, 2013, 68-81.

Alvarenga, L. (2013). Modelagem conceitual e sistemas de organização de conhecimento: uma reflexão baseada em experiências acadêmicas e profissionais no Brasil no domínio da Blblioteconomia e Ciêcnia da Informação. // Dobedei, Vera; Guimarães, José Augusto Chaves (ed.). Complexidade e Organização do Conhecimento: desafi- 
os de nosso século . Rio de Janeiro: ISKO-Brasil ; Marília: FUNDEPE, 2013, 141-146

Alvares Júnior, L. S.; Saldanha, G.S. (2013). O mundo é o assunto - epistemologia da indexação no século XIX. // Ribeiro, Fernanda; Cerveira, Maria Elisa. (ed.). Informação e/ou Conhecimento: as duas faces de Jano. Porto: Faculdade de Letras da Universidade do Porto/ CETAC.MEDIA, 2013, 82-98.

Andrade, I. A. de; Junior, D. W. B.; Cervantes, B. M. N.; Rodrigues, M. R. A. (2011). Organização e representação do conhecimento e os mapas conceituais. // Guimarães, José Augusto Chaves; Dobedei, Vera. (ed.). Desafios e Perspectivas Cientificas para a organização e representação do conhecimento. Marilia: ISKO-Brasil: FUNDEPE, 2011, 83-90.

Arsenault, C.; Widad Mustafa El Hadi. (2012). Dynamism and stability in knowledge organization: from one conference to another Toronto 2000, Lille 2011. // Hadi, Widad Mustafa El (ed.). Knowledge Organization: dynamism et stabilité. Lille: Lavoiser, 2011, 21-31

Bardin, L. (2009). Análise de Conteúdo. Lisboa, Portugal; Edições 70, LDA, 2009.

Barite, M. (2011). La producción científica en Organización del Conocimiento vista através de la Sección Literatura de la revista Knowledge Organization: invisibilidad y transparencia. // Guimarães, José Augusto Chaves; Dobedei, Vera. (ed.). Desafios e Perspectivas Cientificas para a organização e representação do conhecimento. Marilia: ISKO-Brasil: FUNDEPE, 2011, 262-272.

Brascher, M. (2011). Visibilidade científica e interlocução internacional em organização e representação do Conhecimento. // José Augusto Chaves Guimarães; Vera Dobedei (Org.). Desafios e Perspectivas Científicas para a organização e representação do conhecimento. 1ed. Marilia: ISKO-Brasil: FUNDEPE, 2011, p.234-237.

Bufrem, L. S. (2004). Levantando significações para significantes: da gestão do conhecimento a organização do saber. // Encontros Bibli: Revista Eletrônica de Biblioteconomia e Ciência da Informação. 9, n. esp.(del), $1^{\circ}$ sem. http://www.encontros-bibli.ufsc.br/bibesp/esp_01/1 bufrem.pdf.

Café, L. M. A.; Agustín lacruz, M. C.; Barros, C. M. (2011). Organização do Conhecimento: análise conceitual. // Péres Pais, Maria Carmem; Bonome, María G. (ed.). Actas del $X$ Congreso de ISKO-ESPANHA - 20 years of ISKO Spanish Chapter. Universidad da Coruña - Serviço de Publicaciones, 2011, 283-302.

Couzinet, V. (2011). L' organization des connaissances au regard des sciences de l' information et de la communication, une excepetion française?. // Hadi, Widad Mustafa El (ed.). Knowledge Organization: dynamism et stabilité. Lille: Lavoiser, 2011, 35-50.

Dahlberg, I. (1993). Knowledge organization: its scope and possibilities. // Knowledge Organization. 20:4, 211-222.

Dahlberg, I. (2008). The Information Coding Classification (ICC): A modern theory-basead fully-faceted, universal system of knowledge field. // Axiomathes. 18:2, 161-176.

Dal'evedore, P. R.; Almeida, C. C.; Fujita, M. S. L. (2013). A Interpretação de conceitos no processo de indexação aspectos e reflexões semióticas. // Ribeiro, Fernanda; Cerveira, Maria Elisa. (ed.). Informação e/ou Conhecimento: as duas faces de Jano. Porto: Faculdade de Letras da Universidade do Porto/ CETAC.MEDIA, 2013, 190-203.

DODEBEI, V. Pesquisa em organização e representação do conhecimento no Brasil: Uma análise dos cursos de pós-graduação. // José Augusto Chaves Guimarães; Vera Dobedei. (Org.). Desafios e Perspectivas Científicas para a organização e representação do conhecimento. 1ed.Marilia: ISKO-Brasil: FUNDEPE, 2011, p.165-170.
Fox, M. J. (2011) Protoype theory: an alternative concpt theory for categorizing sex and gender. // Proceedings from North American Symposium on Knowledge Organization -NASKO. Toronto, Canada, 2011, 13-22.

Fox, M.; Reece, A. (2013). The impossible decision: social tagging and Derridas's desconstructed hospotality. // The Fourth North American Symposium on Knowledge Organization - NASKO, 2013, Milwaulkee. Transition Cultures, Transition KO: Evolving Exploration, Critical Reflection, and Practical Work, 2013, 58-74.

Frické, Martin (2013). Logic and the organization of information: an introduction. // The Fourth North American Symposium on Knowledge Organization - NASKO, 2013, Milwaulkee. Transition Cultures, Transition KO: Evolving Exploration, Critical Reflection, and Practical Work, 2013, 75-80.

Fujita, M.S.L. (2013). A importância teórica e prática da indexação na fundamentação científica da organização e representação do conhecimento. // Dobedei, Vera; Guimarães, José Augusto Chaves (ed.). Complexidade e Organização do Conhecimento: desafios de nosso século. Rio de Janeiro: ISKO-Brasil ; Marília: FUNDEPE, 2013, 147-159.

Gnoli, C. (2011). Des métadonnées représentant quoi?. // Hadi, Widad Mustafa El (ed.). Knowledge Organization: dynamism et stabilité. Lille: Lavoiser, 2011, 51-66.

Guimarães, José Augusto Chaves. A dimensão teórica do tratamento temático da informação e suas interlocuções com o universo científico da International Society for Knowledge Organization (ISKO). Revista IberoAmericana de Ciência da Informação, - cidade -, v. 1, n. 1, 2008.

Guimarães, J. A. C.; Sales, Rodrigo de; Martínez-Ávila, Daniel; Fernandes Alencar, Maíra (2014). The conceptual dimension of knowledge organization in the ISKO proceedings domain: a Bardinian analysis. // Babik, Wieslaw. (ed.) Knowledge organization in the 21st century: between historical patterns and future perspectives. Wurzburg: Ergon Verlag, 2014, 101-106.

Guimarães, J. A. C.; Ferreira, G. M.; Freitas, M. F. M. (2011). Correntes teóricas do tratamento temático da informação: uma análise de domínio da presença da catalogação de assunto e da indexação nos congressos de ISKO-España. // Péres Pais, Maria Carmem; Bonome, María G. (ed.). Actas del $X$ Congreso de ISKOESPANHA - 20 years of ISKO Spanish Chapter. Universidad da Coruña - Serviço de Publicaciones, 2011, 181194.

Hjørland, B. (2002). Domain analysis in information Science: eleven approaches traditional as well as innovative. // Journal of Documentation. 58:4, 422-462.

Hjørland, B. (2004). Domain analysis: a socio-cognitive orientation for Information Science research. // Bulletin of the American Society for Information Science and Technology. 30:3. http://www.asis.org/Bulletin/Feb04/hjorland.html.

Hjørland, B.; Albrechtsen, H. (1995). Toward a new horizon in information science: domain-analysis. // Journal of the American Society for Information Science. 46:6, 400425.

Jacob, E. K.; Zhang, G. (2013). The role of virtual boundaries in knowledge and organization. // The Fourth North American Symposium on Knowledge Organization NASKO, 2013: Transition Cultures, Transition KO: Evolving Exploration, Critical Reflection, and Practical Work, Milwaulkee. Proceedings from North American Symposium on Knowledge Organization. Milwaukee: University of Wisconsin-Milwaukee, 2013, 127-135

Maccall, S. L. (2011). Knowledge Organization under digital inversion: A theory for cooperative librarian organizing 
practices for online textual artifacts. // Proceedings from North American Symposium on Knowledge Organization -NASKO. Toronto, Canada, 2011, v. 3, p. 13-22.

Marchese, C.; Smiraglia, R. P. (2013). Boundary objects: CWA, an HR firm, and emergent vocabulary. // The Fourth North American Symposium on Knowledge Organization - NASKO, 2013: Transition Cultures, Transition KO: Evolving Exploration, Critical Reflection, and Practical Work, Milwaulkee. Proceedings from North American Symposium on Knowledge Organization. Milwaukee: University of Wisconsin-Milwaukee, 2013, 136142

Martins, G. K.; Moraes, J. B. E. (2013) Sistematização de conteúdos em Organizaçã e Representação do Conhecimento - um estudos nos periódicos de Ciência da Informação no Brasil. // Ribeiro, Fernanda; Cerveira, Maria Elisa (ed.). Informação e/ou Conhecimento: as duas faces de Jano. Porto: Faculdade de Letras da Universidade do Porto/ CETAC.MEDIA, 2013, 421-436.

Miranda, M. L. C.; Paranhos, J. P. B.; Oliveira, J. X.; Paes, M. S. (2011). Organização e representação do conhecimento em religiões yorubanas na Library of Congress Subject Headings. // Guimarães, José Augusto Chaves; Dobedei, Vera. (ed.). Desafios e Perspectivas Cientificas para a organização e representação do conhecimento. Marilia: ISKO-Brasil: FUNDEPE, 2011, 153-159.

Moya-Anegón, F.; Herrero-Solana, V. (2001). Análisis de dominio de la revista mexicana de investigación bibliotecológica. // Información, cultura y sociedad, 5, 10-28.

Murguia, E. I.; Sales, R. (2013). Práticas discursivas na Organização do Conhecimento - Otlet, Bliss e ISKO. // Ribeiro, Fernanda; Cerveira, Maria Elisa. (ed.). Informação e/ou Conhecimento: as duas faces de Jano. Porto: Faculdade de Letras da Universidade do Porto/ CETAC.MEDIA, 2013, 446-461.

Ohly, H.P. (2011a). ISKO - Knowledge Organization in a Changing World. Challeges, Programs, and Mission. // Guimarães, José Augusto Chaves; Dobedei, Vera. (ed.). Desafios e Perspectivas Cientificas para a organização e representação do conhecimento. Marilia: ISKO-Brasil: FUNDEPE, 2011. 238-248

Ohly, H. P. (2011b). Organization, Management and Engineering of Knowlegde: Rivals or Complements? // Péres Pais, Maria Carmem; Bonome, María G. (ed.). Actas del $X$ Congreso de ISKO-ESPANHA - 20 years of ISKO Spanish Chapter. Universidad da Coruña - Serviço de Publicaciones, 2011. 541-551.

Olson, H. A.; Howarth, L. C. A (2013). Space of Transition: Rethinking Surrogates. // The Fourth North American Symposium on Knowledge Organization - NASKO, 2013: Transition Cultures, Transition KO: Evolving Exploration, Critical Reflection, and Practical Work, Milwaulkee. Proceedings from North American Symposium on Knowledge Organization. Milwaukee: University of Wisconsin-Milwaukee, 2013,.161-166

Ortega, C. D. (2013). Aspectos teóricos, procedimentos, normativos e pragmáticos como categorias para uma epistemoçogia da organização da informação. // Dobedei, Vera; Guimarães, José Augusto Chaves (ed.). Complexidade e Organização do Conhecimento: desafios de nosso século . Rio de Janeiro: ISKO-Brasil ; Marília: FUNDEPE, 2013. 34-38.

Pajek (2009). Introductory social network analysis with Pajek. School of Information- University of Michigan. http://ocw.mit.edu/courses/economics/14-15j-networksfall-2009/assignments/MIT14_15JF09_pajek.pdf.

Pattuelli, M. C. (2011). Mapping subjectivity: performing people-centered vocabulary alignment. // Proceedings from North American Symposium on Knowledge Organization-NASKO. Toronto, Canada, 2011. 13-22
Sales, R. Guimarães, J. A. C.. (2013). Contributions of Julius Otto Kaiser to the theoretical framework of Knowledge Organization: basis for the analytical-synthetic method. // The Fourth North American Symposium on Knowledge Organization - NASKO, 2013: Transition Cultures, Transition KO: Evolving Exploration, Critical Reflection, and Practical Work, Milwaulkee. Proceedings from North American Symposium on Knowledge Organization. Milwaukee: University of Wisconsin-Milwaukee, 2013, 167175.

Sanchéz, A. F. G.; Blázquez, B. B.; Rodríguez, I. A. (2011). Análisis bibliométricos de artículos relacionados con la Organización del Conocimiento en revistas españolas recogidas en la Web os Science. // Péres Pais, Maria Carmem; Bonome, María G. (ed.). Actas del X Congreso de ISKO-ESPANHA - 20 years of ISKO Spanish Chapter. Universidad da Coruña - Serviço de Publicaciones, 2011. 171-180.

Schatz, J. S.-S. Nadia; Mills, S. Loehrlein; Aaron , J. (2013). Bibliographic Induction: How KO Systems Optimize Browsing by Supporting Library Users' Prior Knowledge. // The Fourth North American Symposium on Knowledge Organization - NASKO, 2013: Transition Cultures, Transition KO: Evolving Exploration, Critical Reflection, and Practical Work, Milwaulkee. Proceedings from North American Symposium on Knowledge Organization. Milwaukee: University of Wisconsin-Milwaukee, 2013. 176186

Semidão, R. A. M.; Almeida, C. C.; Moreira, W. (2013). Diretrizes para análise conceitual - as perspectivas de Hjorland, Dahlberg e Lakoff. // Ribeiro, Fernanda; Cerveira, Maria Elisa. (ed.). Informação e/ou Conhecimento: as duas faces de Jano. Porto: Faculdade de Letras da Universidade do Porto/ CETAC.MEDIA, 2013, p.589602.

Smiraglia, R. P. (2012). Epistemology of Domain Analysis. // Lee, H-L.; Smiraglia, R.P. (ed.). Cultural frames of knowledge. Wurzburg: Ergon, 2012.111-124

Smiraglia, R. P. (2013). The Epistemological Dimension of Knowledge Organization. // Dobedei, Vera; Guimarães, José Augusto Chaves (ed.). Complexidade e Organização do Conhecimento: desafios de nosso século. Rio de Janeiro: ISKO-Brasil; Marília: FUNDEPE, 2013.17-25.

Sosinska-Kalata, B. (2011) Evolution des sustémes d' organization des connaissances et établissement de critéres pour leur évaluation praxiologique?. // Hadi, Widad Mustafa El (ed.). Knowledge Organization: dynamism et stabilité. Lille: Lavoiser, 2011. 21-31

Tennis, J. T. (2003). Two Axes of Domain Analysis. // Knowledge Organization. 30:3/4, 191-195.

Tognoli, N. B.; Guimarães, J. A. C.; Tennis, J. T. (2013). Transition Cultures, Transition KO: Evolving Exploration, Critical Reflection, and Practical Work. // The Fourth North American Symposium on Knowledge Organization - NASKO, 2013: Transition Cultures, Transition KO: Evolving Exploration, Critical Reflection, and Practical Work, Milwaulkee. Proceedings from North American Symposium on Knowledge Organization. Milwaukee: University of Wisconsin-Milwaukee, 2013. 217-225

Tognoli, N. B.; Milani, S. O.; Barros, T. H. B. (2011). International Society for Knowledge Organization(ISKO): identificação de redes sociais a partir dos Anais de seus eventos (1991-2010). // Péres Pais, Maria Carmem; Bonome, María G. (ed.). Actas del X Congreso de ISKOESPANHA - 20 years of ISKO Spanish Chapter. Universidad da Coruña - Serviço de Publicaciones, 2011. 145158.

Urbizagástegui Alvarado, R. (2009). Elitismo na literatura sobre a produtividade dos autores. // Ciência da Informação, 38:2, http://dx.doi.org/10.1590/S010019652009000200006. 
Varela, A. V.; Barbosa, M. L. A. (2013). O caráter técnico, social, e mediador da organização do conhecimento. // The Fourth North American Symposium on Knowledge Organization - NASKO, 2013: Transition Cultures, Transition KO: Evolving Exploration, Critical Reflection, and Practical Work, Milwaulkee. Proceedings from North American Symposium on Knowledge Organization. Mil- waukee: University of Wisconsin-Milwaukee, 2013. 277282.

Enviado: 2015-04-16. Segunda versión: 2015-06-16. Aceptado: 2015-07-02. 